Fixed Point Theory, 22(2021), No. 1, 3-14

DOI: $10.24193 /$ fpt-ro.2021.1.01

http://www.math.ubbcluj.ro/ nodeacj/sfptcj.html

\title{
BEST PROXIMITY THEOREMS OF PROXIMAL MULTIFUNCTIONS
}

\author{
REZA AHMADI*, ASADOLLAH NIKNAM** AND MAJID DERAFSHPOUR*** \\ * Department of Mathematics, Mashhad Branch, \\ Islamic Azad University, Mashhad, Iran, \\ E-mail: problem.math@hotmail.com \\ *** Department of Mathematics, Mashhad Branch, \\ Islamic Azad University, Mashhad, Iran, \\ E-mail: niknam@um.ac.ir \\ (Corresponding author) \\ *** Department of Mathematics, Mashhad Branch, \\ Islamic Azad University, Mashhad, Iran, \\ E-mail: derafshpour.m@gmail.com
}

\begin{abstract}
Best proximity point theorems for self multifunctions have been proved with different conditions on the space and the considered mappings. In this paper, we prove some best proximity point theorems for a class of generalized multifunctions, namely proximal multifunctions.

Key Words and Phrases: Best proximity point, proximal multifunctions of first kind, proximal multifunctions of second kind, approximatively compact, cyclically Cauchy sequence, fairly Cauchy sequence, fairly complete space, uniform approximation, $T$-approximation, quasi-continuous.

2020 Mathematics Subject Classification: 47H10, 41A65, 90C30.
\end{abstract}

Acknowledgment. The authors thankful to the anonymous referees for the useful suggestions and remarks that contributed to the improvement of the manuscript.

\section{REFERENCES}

[1] A. Abkar, N. Moezzifar, A. Azizi, N. Shahzad, Best proximity point theorems for cyclic generalizaed proximal contractions, Fixed Point Theory Appl., 66(2016).

[2] C.D. Aliprantis, K.C. Border, Infinite Dimensional Analysis: A Hitchhiker's Guide, Third Edition, Springer-Verlag Berlin Heidelberg, 2006.

[3] M.A. Al-Thagafi, N. Shahzad, Convergence and existence for best proximity points, Nonlinear Anal., 70(2009), no. 10, 3665-3671.

[4] M. Benchohra, J. Henderson, S. Ntouyas, Impulsive Differential Equations and Inclusions, Hindawi Publishing Corporation., 2, New York, 2006.

[5] G. Debreu, Theory of Value, Wiley, New York, 1959.

[6] M. Derafshpour, Sh. Rezapour, Picard operators on ordered metric spaces, Fixed Point Theory, 15(2014), no. 1, 59-66.

[7] C. Di Bari, T. Suzuki, C. Vetro, Best proximity points for cyclic Meir-Keeler contractions, Nonlinear Anal., 69(2008), no. 11, 3790-3794. 
[8] A.A. Eldered, P. Veeramani, Existence and convergence of best proximity points, J. Math. Anal. Appl., 323(2006), no. 2, 1001-1006.

[9] K. Fan, Extensions of two fixed point theorems of F.E. Browder, Math. Z., 112(1969), 234-240.

[10] A. Fernandez-Leon, M. Gabeleh, Best proximity pair theorems for noncyclic mappings in Banach and metric spaces, Fixed Point Theory, 17(2016), no. 1, 63-84.

[11] E. Karapinar, F. Khojasteh, An approach to best proximity points results via simulation functions, J. Fixed Point Theory Appl., 19(2017), 1983-1995.

[12] K. Khammahawong, P. Kumam, D.M. Lee, Y.J. Cho, Best proximity points for multi-valued Suzuki $\alpha$-F-proximal contractions, J. Fixed Point Theory Appl., 19(2017), 2847-2871.

[13] W.A. Kirk, S. Reich, P. Veeramani, Proximal retracts and best proximity pair theorems, Numer. Funct. Anal. Optim., 24 (2003), no. 7-8, 851-862.

[14] E.N. Mahmudov, Approximation and Optimization of Discrete and Differential Inclusions, Burlington, MA, Elsevier, 2011.

[15] S. Nadler, Multivalued contraction mappings, Pacific. J. Math., 30(1969), no. 2, 475-488.

[16] V. Pragadeeswara, M. Marudai, P. Kumam, Best proximity point theorems for multivalued mappings on partially ordered metric spaces, J. Nonlinear Sci. Appl., 9(2016), 1911-1921.

[17] S. Sadiq Basha, Extensions of Banach's contraction principle, Numer. Funct. Anal. Optim., 31(2010), no. 5, 569-579.

[18] S. Sadiq Basha, Best proximity points: optimal solutions, J. Optim. Theory. Appl., 151(2011), 210-216.

[19] S. Sadiq Basha, Best proximity point theorems in the frameworks of fairly and proximally complete spaces, J. Fixed Point Theory Appl., 19(2017), 1939-1951.

[20] S. Sadiq Basha, N. Shahzad, Best proximity point theorems for generalized proximal contraction, Fixed Point Theory Appl., 42(2012).

[21] S. Sadiq Basha, N. Shahzad, C. Vetro, Best proximity point theorems for proximal cyclic contraction, J. Fixed Point Theory Appl., 19(2017), 2647-2661.

[22] S. Sadiq Basha, P. Veeramani, Best approximations and best proximity pairs, Acta Sci. Math. (Szeged), 63(1997), 289-300.

Received: October 18, 2018; Accepted: March 27, 2020. 